\title{
Doppler assessment of hypoxic pulmonary vasoconstriction and susceptibility to high altitude pulmonary oedema
}

\author{
J L Vachiéry, T McDonagh, J J Moraine, J Berré, R Naeije, H Dargie, A J Peacock
}

\begin{abstract}
Background - Subjects with previous high altitude pulmonary oedema may have stronger than normal hypoxic pulmonary vasoconstriction. Susceptibility to high altitude pulmonary oedema may be detectable by echo Doppler assessment of the pulmonary vascular reactivity to breathing a hypoxic gas mixture at sea level.
\end{abstract}

Methods - The study included 20 healthy controls, seven subjects with a previous episode of high altitude pulmonary oedema, and nine who had successfully climbed to altitudes of $6000-8842 \mathrm{~m}$ during the 40th anniversary British expedition to Mount Everest. Echo Doppler measurements of pulmonary blood flow acceleration time (AT) and ejection time (ET), and of the peak velocity of the tricuspid regurgitation jet (TR), were obtained under normobaric conditions of normoxia (fraction of inspired oxygen, $\left.\mathrm{FIO}_{2}, 0 \cdot 21\right)$, of hyperoxia $\left(\mathrm{FIO}_{2} 1 \cdot 0\right)$, and of hypoxia $\left(\mathrm{FIO}_{2} 0 \cdot 125\right)$.

Results - Hypoxia decreased AT/ET by mean (SE) $0.06(0.01)$ in the control subjects, by $0.11(0.01)$ in those susceptible to high altitude pulmonary oedema, and by $0.02(0.02)$ in the successful high altitude climbers. Hypoxia increased $T R$ in the three groups by $0.22(0.06)(n=14), 0.56$ $(0 \cdot 13)(\mathrm{n}=5)$, and $0.18(0 \cdot 1)(\mathrm{n}=7) \mathrm{m} / \mathrm{s}$, respectively. However, AT/ET and/or TR measurements outside the normal range, defined as mean \pm 2 SD of measurements obtained in the controls under hypoxia, were observed in only two of the subjects susceptible to high altitude pulmonary oedema and in five of the successful high altitude climbers.

Conclusions - Pulmonary vascular reactivity to hypoxia is enhanced in subjects with previous high altitude pulmonary oedema and decreased in successful high altitude climbers. However, echo Doppler estimates of hypoxic pulmonary vasoconstriction at sea level cannot reliably identify subjects susceptible to high altitude pulmonary oedema or successful high altitude climbers from a normal control population.

(Thorax 1995;50:22-27)

Keywords: Doppler, hypoxic pulmonary vasoconstriction, high altitude pulmonary oedema.
High altitude pulmonary oedema is an uncommon but severe complication of acute mountain sickness that occurs in non-acclimatised individuals exposed to altitudes higher than 2000-3000 m. ${ }^{12}$ Although the pathogenesis of high altitude pulmonary oedema is still disputed, one theory relates the condition to excessive hypoxic pulmonary vasoconstriction (HPV). ${ }^{12}$ Support for this theory has come from reports of enhanced $\mathrm{HPV}^{3-8}$ and very high pulmonary artery pressure in subjects with a previous history of high altitude pulmonary oedema, or during an episode. ${ }^{49-13}$ This theory is also supported by the effectiveness in the treatment of high altitude pulmonary oedema ${ }^{1213}$ of nifedipine, a calcium channel blocker which inhibits HPV in humans, ${ }^{14}$ and the experimental demonstration of stress failure in pulmonary capillaries. ${ }^{15}$ The incidence of high altitude pulmonary oedema within 24 hours of a rapid ascent to $4559 \mathrm{~m}$ is about $10 \%$, but increases to $60 \%$ in subjects with a previous episode of high altitude pulmonary oedema. ${ }^{13}$ Thus, there appears to be a constitutional susceptibility to high altitude pulmonary oedema which may relate to a more vigorous pulmonary vascular reaction to hypoxia.

Recent advances in echo Doppler technology have allowed remarkable improvements in the non-invasive evaluation of the pulmonary circulation. ${ }^{16-20}$ The severity of pulmonary hypertension can now be estimated from the peak velocity of the tricuspid regurgitation jets (TR), or from the shape of pulmonary flow-velocity curves. ${ }^{1920}$ This approach has already been used for the non-invasive study of the effects of changes in inspired oxygen on pulmonary haemodynamics. ${ }^{132122}$

The purpose of the present study was to investigate whether susceptibility to high altitude pulmonary oedema, or tolerance to high altitudes, might be identified by echo Doppler indices of HPV at sea level.

\section{Methods}

SUBJECTS

Seven subjects who had had a previous episode of high altitude pulmonary oedema, 20 unselected healthy controls, and nine controls who had successfully climbed to altitudes of $6000-8848 \mathrm{~m}$ gave informed consent to the study which was approved by the ethical committees of the Erasme University Hospital, Brussels and of the Western Infirmary, Glas- 
gow. All had normal physical examinations, chest radiographs, and electrocardiograms. The subjects with a susceptibility to high altitude pulmonary oedema and the unselected controls were investigated in Brussels. The 40th anniversary British expedition to Mount Everest offered the opportunity to investigate a control group in Glasgow consisting of successful high altitude climbers.

The subjects with previous high altitude pulmonary oedema comprised six men and one woman of mean (SD) age 38 (10) years (range 25-58) who had experienced high altitude pulmonary oedema at altitudes ranging from $3180 \mathrm{~m}$ to $5900 \mathrm{~m}$ at various locations. The diagnosis of high altitude pulmonary oedema was based on the following clinical criteria ${ }^{12}$ : dyspnoea at rest or slight effort, nocturnal dyspnoea, orthopnoea, cough with or without haemoptysis, and pulmonary crackles in the context of classical symptoms of acute mountain sickness (headache, nausea, insomnia), appearing within hours after a rapid ascent to an altitude above $3000 \mathrm{~m}$ and improving rapidly after returning to a lower altitude. The period between the episode of high altitude pulmonary oedema and the study ranged from four months to eight years.

The unselected controls were 11 men and nine women of mean (SD) age 27 (5) years (range 21-39) residing in Belgium and employed as nurses, physical therapists, or physicians at the Erasme University Hospital. None had ever climbed or travelled to altitudes higher than $3000 \mathrm{~m}$.

The successful high altitude climbers consisted of eight men and one woman of mean (SD) age 43 (7) years (range 30-63), all of

Echo Doppler indices of pulmonary haemodynamics in 20 normal controls, seven subjects with previous high altitude pulmonary oedema (HAPO), and nine successful high altitude climbers breathing different concentrations of inspired oxygen

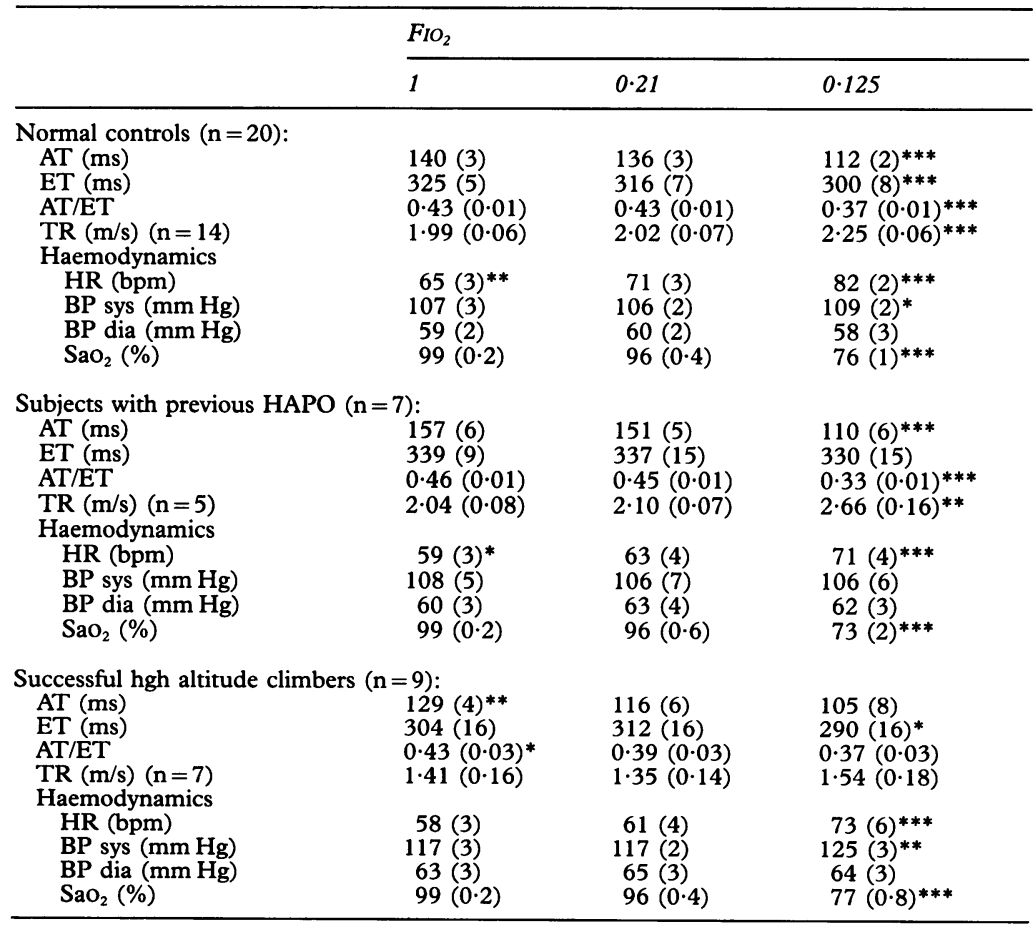

$\mathrm{FIO}_{2}=$ fraction of inspired oxygen; $\mathrm{AT}=$ pulmonary blood flow acceleration time; $\mathrm{ET}=$ right ventricular ejection time; $T R=$ peak velocity of tricuspid regurgitation jet; $H R=$ heart rate; $B P$ ventricular ecetion time; $\mathrm{TR}=$ peak velocity of tricuspid regurgitation jet; $\mathrm{HR}=$ heart rate; $\mathrm{BP}$
${ }^{*} \mathrm{p}<0.05,{ }^{* *} \mathrm{p}<0 \cdot 01,{ }^{* * *} \mathrm{p}<0.001 \mathrm{FIO}_{2}$ of 1 or $0.125 v 0 \cdot 21$. whom had successfully climbed to altitudes of $4000-8200 \mathrm{~m}$. None showed symptoms of high altitude pulmonary oedema nor any change in spirometric indices during the climb.

\section{MEASUREMENTS}

The echo Doppler examinations were performed using a Hewlett-Packard SONOS 1000 ultrasound system with a combined $3.5 \mathrm{MHz}$ two-dimensional imaging/Doppler transducer. Doppler recordings were obtained from the parasternal short axis or apical position with each subject lying with a slight left oblique rotation. Colour mode Doppler was used to superimpose the ultrasound beam on the flowvelocity axis to avoid any angle correction. The pulsed Doppler mode was used to study pulmonary flow velocity. The sample volume (size $3 \times 3 \mathrm{~mm}$ ) was located in the central part of the pulmonary trunk or in the right ventricular outflow tract, close to the valve. Acceleration time (AT) was defined as the interval between the onset of ejection and the peak flow velocity. Right ventricular ejection time (ET) was defined as the time between the onset of ejection to that of zero flow velocity. The maximum velocity of the tricuspid regurgitation jet (TR) was measured by continuous wave Doppler imaging. The echo Doppler examinations were recorded and cross-checked by the cardiologists of the Brussels and Glasgow teams.

Blood pressure was measured using an automated oscillometric device (Accutorr TM 1A, Datascope Corp, Paramus, New Jersey, USA). Heart rate was determined from a continuously monitored electrocardiographic lead. Arterial oxygen saturation $\left(\mathrm{SaO}_{2}\right)$ was measured continuously by pulse oximetry (Hewlett-Packard or Ohmeda).

\section{STUDY PROTOCOL}

Echo Doppler measurements were performed in triplicate when the subjects were in a steady state as evaluated by stable heart rate, blood pressure, and $\mathrm{SaO}_{2}$ for at least 10 minutes at each of the following fractions of inspired oxygen $\left(\mathrm{FIO}_{2}\right): 1,0 \cdot 21$, and $0 \cdot 125$. Gases were administered by a face mask in random order.

\section{DATA ANALYSIS}

All values are expressed as mean (SE). The effects of changes in $\mathrm{FIO}_{2}$ were evaluated using a three-factor repeated measure analysis of variance. When the $\mathrm{F}$ ratio of the analysis of variance reached a critical $\mathrm{p}<0.05$ value, modified $t$ tests with an adjustment for multiple comparisons were used to compare specific situations. $^{23}$

\section{Results}

BASELINE MEASUREMENTS BREATHING ROOM AIR Compared with controls, subjects with a susceptibility to high altitude pulmonary oedema had an increased AT and ET and a decreased heart rate $(p<0 \cdot 05)$, while successful high al- 


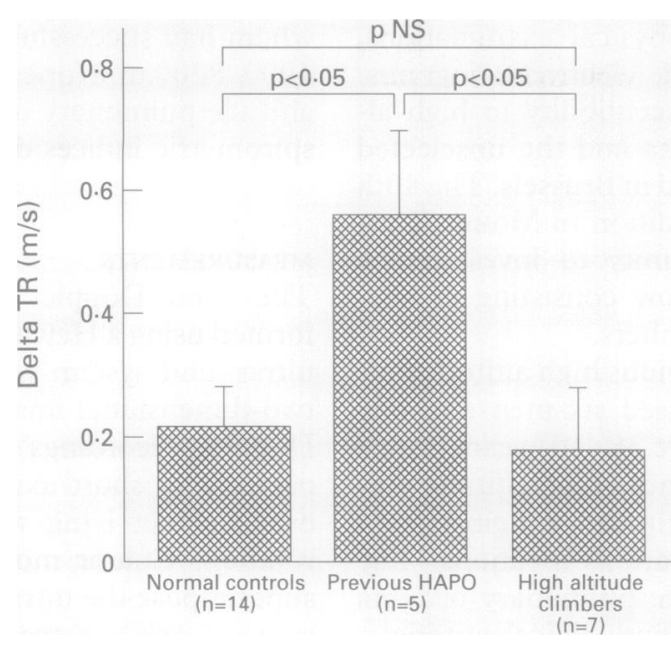

Figure 1 Hypoxia-induced increases in maximum velocity of tricuspid regurgitation jets (TR) calculated as TR measured at an $\mathrm{FIO}_{2}$ of $0 \cdot 125$ minus TR measured at an $\mathrm{FIO}_{2}$ of 0.21 (delta TR) in normal controls, in subjects with previous high altitude pulmonary oedema (HAPO) and in successful high altitude climbers. Subjects with previous high altitude pulmonary oedema had an increased hypoxic response compared with the other groups.

titude climbers had decreased AT, heart rate, and TR, and an increased systolic blood pressure $(p<0.05)$ (table). There were no other differences in baseline variables between the three groups.

EFFECTS OF CHANGES IN $\mathrm{FIO}_{2}$

In the control subjects hyperoxia decreased heart rate, while hypoxia decreased AT, ET, $\mathrm{AT} / \mathrm{ET}$, and $\mathrm{SaO}_{2}$ and increased TR, heart rate, and systolic blood pressure (table). In the subjects susceptible to high altitude pulmonary oedema hyperoxia decreased heart rate while hypoxia decreased AT, ET, AT/ET, and $\mathrm{SaO}_{2}$ and increased TR and heart rate (table). In the successful high altitude climbers hyperoxia increased AT and AT/ET, while hypoxia increased heart rate and systolic blood pressure

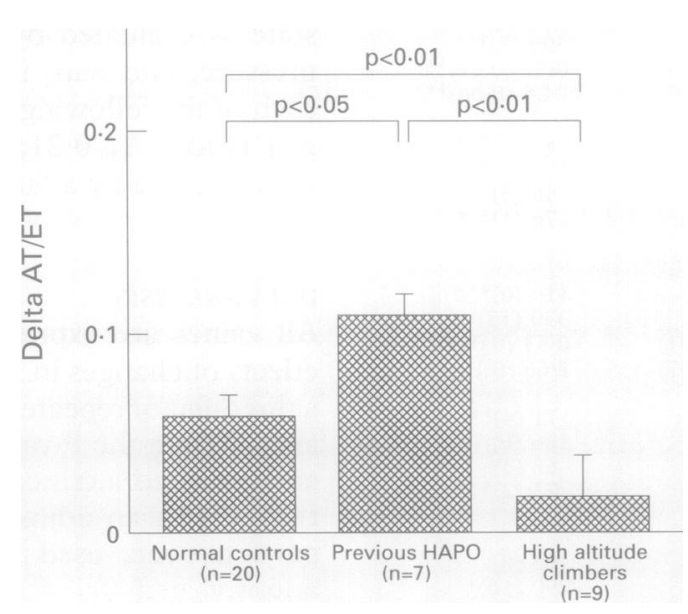

Figure 2 Hypoxia-induced decreases in pulmonary blood flow acceleration time $(A T)$ on right ventricular ejection time (ET) calculated as $A T / E T$ at an $\mathrm{FIO}_{2}$ of 0.21 minus $A T / E T$ at an $\mathrm{FIO}_{2}$ of 0.125 (delta $A T / E T$ ) in normal controls, in subjects with previous high altitude pulmonary oedema (HAPO), and in successful high altitude climbers The hypoxic response was increased in subjects with previous high altitude pulmonary oedema and decreased in successful high altitude climbers.

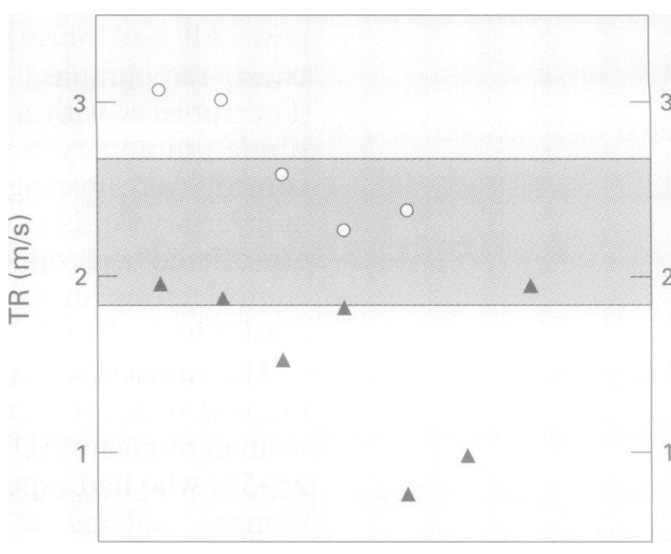

Figure 3 Maximum velocity of tricuspid regurgitation jets (TR) in hypoxia in subjects with previous high altitude pulmonary oedema (HAPO) (O) and in successful high altitude climbers ( $\mathbf{\Delta})$. The shaded area represents limits of normal calculated from mean $\pm 2 S D$ of measurements in 14 controls. Two of five subjects with previous high altitude pulmonary oedema had greater than normal hypoxic TR, and four of seven high altitude climbers had lower than normal hypoxic TR.

and decreased $\mathrm{SaO}_{2}$ and ET, but did not affect AT, AT/ET or TR (table).

\section{HYPOXIA-INDUCED CHANGES IN PULMONARY HAEMODYNAMICS}

The hypoxia-induced increase in TR was similar in controls and successful high altitude climbers, but was enhanced in subjects susceptible to high altitude pulmonary oedema compared with the two other groups (fig 1). The hypoxia-induced decrease in AT/ET was increased in subjects susceptible to high altitude pulmonary oedema compared with controls, and decreased in successful high altitude climbers (fig 2). Two of the five subjects susceptible to high altitude pulmonary oedema and three of the seven successful high altitude climbers in whom the measurements could be obtained had a TR outside the normal range (defined as mean $\pm 2 \mathrm{SD}$ of the measurements

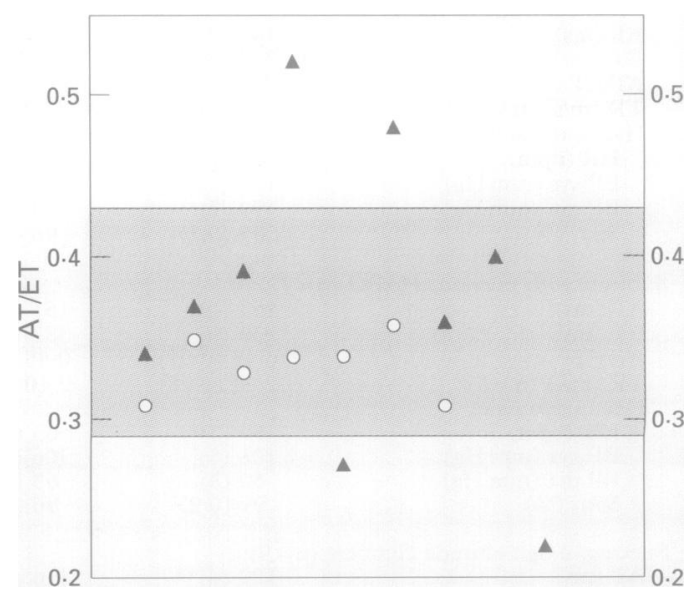

Figure 4 Ratio of pulmonary blood flow acceleration time (AT) to right ventricular ejection time (ET) in hypoxia in subjects with previous high altitude pulmonary oedema (HAPO) (O) and in successful high altitude climbers (A). The shaded area represents limits of normal calculated as mean $\pm 2 S D$ of measurements in 20 controls. None of the subjects with previous high altitude pulmonary oedema had lower than normal hypoxic AT/ ET. Successful high altitude climbers had normal, higher than normal, or lower than normal AT/ET. 
obtained in 14 of the normal controls) (fig 3). None of the subjects susceptible to high altitude pulmonary oedema and four of the successful high altitude climbers had an AT/ET value outside the normal range (defined as mean $\pm 2 \mathrm{SD}$ of the measurements, obtained in the 20 controls) (fig 4).

\section{Discussion}

Our results suggest that the pulmonary vascular reactivity to hypoxia is enhanced in subjects with previous high altitude pulmonary oedema and decreased in successful high altitude climbers. However, echo Doppler estimation of HPV at sea level cannot reliably identify subjects susceptible to high altitude pulmonary oedema, nor successful high altitude climbers, from a general control population.

Several approaches based on echo Doppler technology have been developed for the noninvasive evaluation of pulmonary hypertension. Among these, the calculation of a transtricuspid pressure gradient based on the maximum velocity of triscuspid regurgitation jets ${ }^{17}$ and time to peak velocity measured on a pulmonary flow-velocity curve ${ }^{16}$ have proved most useful clinically. ${ }^{18-20}$ Triscuspid regurgitation jets are recovered in $70-90 \%$ of patients and have been found to correlate well with systolic right ventricular pressure measured at right heart catheterisation with correlation coefficients from 0.87 to $0.97 .{ }^{17-19}$ The recovery rate of TR in subjects without pulmonary hypertension is lower at about $70 \%,{ }^{21}$ as in the present study. Pulmonary flow-velocity curves are recovered in $70-91 \%$ of patients, ${ }^{1619}$ and AT has been found to correlate highly with invasive measurements of mean pulmonary artery pressure with correlation coefficients from -0.65 to $-0 \cdot 85 .^{1618}$ Since AT decreases when heart rate increases, it is understandable that these correlations are improved by adding a correction for heart rate ${ }^{1618}$ - for example, by dividing AT by ET. ${ }^{161920}$ In the present study pulmonary flow-velocity curves could be recovered in all subjects at every value of $\mathrm{FIO}_{2}$.

Based on linear or curvilinear best fits of multipoint plots of TR, AT, or AT/ET versus simultaneous invasive determinations of pulmonary artery pressure or pulmonary vascular resistance, several equations have been proposed for the conversion of the Doppler measurements into pressures or resistances. ${ }^{16-20}$ In spite of the above mentioned good correlations, the predictive value of these equations on an individual basis is relatively poor. This is explained by the many assumptions upon which conversions of Doppler measurements to pressures or resistances are based. The conversion of TR to a systolic pulmonary artery pressure rests on the use of a simplified form of the Bernouilli equation and the clinical evaluation of right atrial pressure. ${ }^{1920}$ The shape of a pulmonary flow-velocity curve is determined not only by pressure, but by a dynamic interplay between volume flow, resistance, elastance, and wave reflections. ${ }^{19}$ We therefore report the actual Doppler data instead of less reliable derived pressures or calculated resistances.
Doppler flow-velocity patterns may be very sensitive to sample location. ${ }^{19}$ Acceleration time and, eventually, AT/ET can be significantly shortened when sampling is done within the pulmonary trunk only a little further away from the pulmonary valve or closer to the posterior pulmonary wall. ${ }^{19} \mathrm{~A}$ careful review of our recordings showed that this was the most likely explanation for the shorter AT and wider scattering of AT/ET in the successful high altitude climbers. This limits any comparison of absolute values of AT and AT/ET between the subjects studied in Glasgow and in Brussels. Small differences in the sampling site probably account for the very low AT/ET values reported in normal subjects close to sea level in other studies. ${ }^{13}$ Indices of pulmonary flow-velocity curves are dependent on the examiner, even when, as in the present study, care is taken to use exactly the same method.

Breathing $100 \%$ oxygen has almost no effect on normal pulmonary artery pressure or vascular resistance. ${ }^{25}$ It is not surprising, therefore, that hyperoxia had no effect on TR in any of our three study groups, nor on AT or AT/ ET in the normal control subjects and those susceptible to high altitude pulmonary oedema. However, $100 \%$ oxygen increased AT and AT/ ET in the successful high altitude climbers. An increase in AT which persisted after correction for decreased heart rate has been reported in normal volunteers studied in Tucson at an altitude of about $1000 \mathrm{~m} .^{22}$ This may be explained by release of a slight degree of HPV at this altitude. In our study oxygen reduced the heart rate in the normal controls and in the subjects susceptible to high altitude pulmonary oedema, but not significantly in the high altitude climbers. Alternatively, the sampling site closer to the posterior wall of the pulmonary artery in the high altitude climbers may have made the flow-velocity curves more sensitive to changes in pulmonary vascular impedance. ${ }^{19}$ Oxygen-induced increases in AT and in AT/ ET in this group could thus be accounted for by subtle decreases in pulmonary vascular elastance and/or reflected waves, which may conceivably occur in the absence of actual changes in pulmonary vascular resistance.

The hypoxia-induced increases in TR and decreases in AT/ET were greater in subjects with previous high altitude pulmonary oedema than controls, in keeping with the hypothesis that susceptibility to high altitude pulmonary oedema is somehow linked to enhanced HPV. ${ }^{12}$ These data agree with some, but not all, previous invasive haemodynamic studies in subjects susceptible to high altitude pulmonary oedema challenged acutely with an $\mathrm{FiO}_{2}$ of $0 \cdot 12-0 \cdot 15$ in normobaric conditions. In one study five men with previous high altitude pulmonary oedema had abnormally high pulmonary artery pressures when a rapid ascent to $3100 \mathrm{~m}$ was combined with prolonged exercise. ${ }^{3}$ However, when tested at sea level only one had an enhanced hypoxic pulmonary pressor response. Furthermore, acute relief of hypoxia at high altitude did not restore pulmonary vascular resistance to normal, suggesting that some factor other than hypoxia 
accounted for the excessive pulmonary hypertension supposedly responsible for pulmonary oedema formation at high altitude. ${ }^{3}$ One of the two subjects with high altitude pulmonary oedema reported by Penazola and Sime was subsequently investigated at sea level and showed a normal increase in pulmonary artery pressures in response to a hypoxic challenge. ${ }^{4}$ In another study 44 Indian soldiers with previous high altitude pulmonary oedema had their hypoxic pulmonary pressor response compared with that of 51 soldiers who had also been on duty in the Himalayan regions at altitudes of $3000-6000 \mathrm{~m}$ but without evidence of high altitude pulmonary oedema. ${ }^{5}$ Susceptibility to high altitude pulmonary oedema was associated with a slightly enhanced hypoxic pulmonary pressor response but the overlap between the groups was considerable and the difference of dubious significance. Seven children with previous high altitude pulmonary oedema showed an increase in mean pulmonary artery pressure from 22 to $56 \mathrm{~mm} \mathrm{Hg}$ breathing a $\mathrm{FIO}_{2}$ of $0 \cdot 16$, compared with a rise from 17 to $19 \mathrm{~mm} \mathrm{Hg}$ in six controls. ${ }^{6}$ However, five of these seven children with previous high altitude pulmonary oedema resided at Leadville $(3060 \mathrm{~m})$ until the day before the hypoxic testing in Denver $(1600 \mathrm{~m})$, so these results are difficult to compare with those of studies performed on adults at sea level. Interestingly, the controls had a surprisingly small hypoxic pressor response. A greater normobaric hypoxia-induced increase in pulmonary artery pressures has been reported in five men with previous high altitude pulmonary oedema at altitudes around $3000 \mathrm{~m}$ compared with five controls who had climbed above this altitude without any symptoms of high altitude pulmonary oedema. ${ }^{7}$ The same was found in eight subjects with previous high altitude pulmonary oedema compared with six successful climbers at altitudes around $3000 \mathrm{~m} .{ }^{8}$ However, normal normobaric hypoxic pulmonary pressor responses have been reported in two subjects with previous high altitude pulmonary oedema compared with a normal unselected control population. $^{2627}$

The magnitude of HPV varies considerably from one species to another and, within a species, from one individual to another. ${ }^{28}$ If the limits of normal human HPV are defined using right heart catheterisation studies performed in 32 healthy volunteers aged $21-42$ years (mean $25)$, it is apparent that most previously reported subjects with a susceptibility to high altitude pulmonary oedema actually had a normal normobaric HPV. ${ }^{2627}$ In the present study only two of the subjects with previous high altitude pulmonary oedema had a TR above normal in hypoxia, and none had a lower than normal AT/ET in hypoxia. However, differences in hypoxic responses were more important with a minimal overlap between subjects susceptible to high altitude pulmonary oedema and successful high altitude climbers. This is in keeping with previous invasive studies in which subjects susceptible to high altitude pulmonary oedema have been compared with successful high altitude climbers or dwellers. ${ }^{6-8}$
Interestingly, participants in the 40th anniversary British expedition to Mount Everest who successfully reached extremely high altitudes had a blunted hypoxic pulmonary pressor response. Very small or absent HPV has been reported previously in some, ${ }^{67}$ but not all, ${ }^{58}$ subjects who went without problem to altitudes of $3000-4000 \mathrm{~m}$. It may be that a blunted HPV is needed for a successful climb to the summit of Mount Everest. Native Tibetans, probably the world's highest and longest dwelling high altitude population, have minimal hypoxic pulmonary hypertension. ${ }^{29}$ Native high altitude cattle present with structural and functional adaptations leading to decreased pulmonary vascular reactivity to hypoxia. ${ }^{30} \mathrm{~A}$ decreased HPV might protect against high altitude pulmonary oedema and perhaps is associated with yet other unknown characteristics essential for a successful acute, as well as chronic, adaptation to altitudes.

Our thanks to Marie-Thérèse Gautier who helped in the preparation of this report, to all the participants in the study, and to the laboratory staff of both hospitals.

Andrew Peacock was supported by the Wellington Foundation (London, UK).

1 Heath D, Williams DR. High-altitude medicine and pathology. London: Butterworths, 1989:121-48

2 Ward MP, Milledge JS, West JB. High altitude medicine and physiology. Philadelphia: University of Pennsylvania Press, 1989.

3 Hultgren HN, Grover RF, Hartley LH. Abnormal circulatory responses to high altitude in subjects with a previous history of high-altitude pulmonary edema. Circulation 1971;44:759-70.

4 Penazola D, Sime F. Circulatory dynamics during high altitude pulmonary edema. Am f Cardiol 1969;23:369-78.

5 Viswanathan R, Jain SK, Subramanian S, Subramanian TAV, Dua GL, Giri J. Pulmonary edema of high altitude. II. Clinical, aerohemodynamic and biochemical studies in a group with history of pulmonary edema of high altitude. Am Rev Respir Dis 1969;100:334-49.

6 Fasules JW, Wiggins JW, Wolfe RR. Increased lung vasoreactivity in children from Leadville, Colorado, after recovery from high-altitude pulmonary edema. Circulation 1985;72:957-62

7 Kawashima A, Kubo K, Kobayashi T, Sekiguchi M. Hemodynamic responses to acute hypoxia, hypobaria, and exercise in subjects susceptible to high-altitude pulmonary edema. F Appl Physiol 1989;67:1982 - 9 .

8 Yagi H, Yamada H, Kokayashi T, Sekiguchi M. Doppler assessment of pulmonary hypertension induced by hypoxic breathing in subjects susceptible to high altitude pulmonary edema. Am Rev Respir Dis 1990;142:796-801.

9 Fred HL, Schmidt AM, Bates T, Hecht HH. Acute pulmonary edema of altitude: clinical and physiologic obmonary edema of altitude: Circulation 1962;25:929-37.

10 Hultgren H, Lopez C, Lundberg E, Miller H. Physiologic studies of pulmonary edema at high altitude. Circulation 1964;29:393-408.

11 Roy SB, Guleria JS, Khanna PK, Machanda SC, Pande JN, Subba PS. Hemodynamic studies in high altitude pulmonary edema. Br Heart $\mathcal{f}$ 1969;31:52-8.

12 Oelz O, Ritter M, Jenni R, Maggiorini M, Waber U, Vock P. Nifedipine for high-altitude pulmonary oedema. Lancet 1989;ii: $1241-4$

13 Bärtsch P, Maggiorini M, Ritter M, Noti C, Vock P, Oelz $O$. Prevention of high-altitude pulmonary edema by nifedipine. N Engl f Med 1991;325:1284-9.

14 Naeije R, Mélot C, Mols P, Hallemans R. Effects of vasodilators on hypoxic pulmonary vasoconstriction in normal man. Chest 1982;82:404-10.

15 West JB, Mathieu-Costello O. Stress failure of pulmonary capillary: role in lung and heart disease. Lancet 1992;340 $762-7$.

16 Kitabatake A, Inoue $M$, Asao M, Masuyama T, Tanouch $\mathrm{J}$, Morita $\mathrm{T}$, et al. Noninvasive evaluation of pulmonary hypertension by a pulsed Doppler technique. Circulation 1983;68:302-9.

17 Yock PG, Popp RL. Noninvasive estimation of right ventricular systolic pressure by Doppler ultrasound in patients with tricuspid regurgitation. Circulation 1984;70:657-62.

18 Chan KL, Currie P, Seward JB, Hagler DJ, Mair DD, Tajik $\mathrm{J}$. Comparison of three Doppler ultrasound methods in the prediction of pulmonary artery pressure. $\mathfrak{F} \mathrm{Am}$ Coll the prediction of pulmo
Candiol 1987;9:549-54.

19 Hatle L, Angelsen B. Doppler ultrasound in cardiology: physical principles and clinical application. 2nd edn. Philadelphia: principles and clinical application. 2nd edn.

20 Feigenbaum $\mathrm{H}$. Hemodynamic information derived from echocardiography. In: Echocardiography. 4th edn. Philadelphia: Lea and Febiger, 1986:188-229. 
21 Peacock AJ, Challenor V, Sutherland G. Estimation of pulmonary artery pressure by Doppler echography in normal subjects made hypoxic. Respir Med 1990;84:335-7.

22 Ernst JH, Goldberg SJ. Do changes in fraction of inspired oxygen affect pulmonary artery acceleration time? $A m \mathcal{F}$ Cardiol 1990;65:252-4

23 Winer BJ. Statistical principles in experimental design. 2nd edn. New York: McGraw-Hill, 1971:514-603.

24 Upward J, Challenor V, Sutherland G. "Physiological" tricuspid and pulmonary regurgitation: their varying incidence in a representative adult population. Br Heart $\mathcal{f}$ 1985;54:618.

25 Mélot C, Dechamps P, Hallemans R, Decroly P, Mols P. Enhancement of hypoxic pulmonary vasoconstriction by low dose of almitrine bismesylate in normal man. Am Rev Respir Dis 1989;139:111-19.

26 Naeije R, Mélot C, Lejeune P. Hypoxic pulmonary vaso- constriction and high altitude pulmonary edema. $\mathrm{Am} \mathrm{Rev}$ Respir Dis 1986;134:332-3.

27 Naeije R, Mélot C. Acute pulmonary oedema on the Ruwenzori. Br Heart $₹$ 1990;64:400-2.

28 Grover RF, Wagner WW, McMurtry IF, Reeves JT. Pulmonary circulation. In: Handbook of physiology. The cardiovascular system. Peripheral circulation and organ blood flow. Bethesda American Physiological Society, 1983:Section 2, Volume III, Part 1, Chapter 4, 103-6.

29 Groves BM, Droma T, Sutton JR, McCullough RG, McCullough RE, Zhuang J, et al. Minimal hypoxic pulmonary hypertension in normal Tibetans at $3658 \mathrm{~m}$. f Appl Physiol 1993;74:312-8.

30 Durmowicz AG, Hofmeister S, Kadyraliev TK, Aldashev AA, Stenmark KR. Functional and structural adaptation of the yak pulmonary circulation to residence at high altitude. F Appl Physiol 1993;74:2276-85. 